\title{
Nonlinear Load and RLC Pulse Shaping Surge Generator Models in Simulation Environment
}

\author{
Mahesh Edirisinghe \\ Department of Physics, University of Colombo, Colombo, Sri Lanka \\ E-mail address:mahesh@phys.cmb.ac.lk
}

\begin{abstract}
The present study was performed in order to understand how a nonlinear load affects the characteristics of the RLC pulse shaping surge generator waveforms under simulation studies. The obtained results were compared when analytical equations were used as source models for both 1.2/50 $\mu$ s voltage \& 8/20 $\mu$ s current impulses. Three circuit models; Capacitor Bank generator, Combination waveform generator and Schaffner generator were evaluated under nonlinear loads varied from $10 \Omega$ to $10^{6} \Omega$ for voltage impulses and for current impulses it was from $10^{-4} \Omega$ to $1 \Omega$. As it can perceive from the analysis, delay time, rise time and FWHM remain unchanged throughout the tested impedance range when used model equations as generator sources. It was found that for voltage impulses, these values were $4.95 \mu \mathrm{s}, 1.20 \mu \mathrm{s}$ and $81.8 \mu$ s respectively. For current impulses, these values were $16.2 \mu \mathrm{s}, 8.02 \mu \mathrm{s}$ and $20.7 \mu$ s respectively. However results obtained for generator circuit models shows that delay time, rise time and FWHM deviated from above values up to maximum of $35.15 \%, 106.67 \% \& 115.16 \%$ respectively for voltage impulses. For current impulses these deviations were up to maximum of $79.63 \%, 48.75 \% \& 39.61 \%$ respectively. The deviations were due to influence caused by the nonlinear load to the generator circuit parameters and effective internal impedance.
\end{abstract}

Keywords: Nonlinear load; surge generator; surge protection; loading effect

\section{INTRODUCTION}

Lightning is one of the major sources of electrical overstresses that can cause failure, permanent degradation, or temporary malfunction of electrical \& electronic devices and systems [1,2]. For example, insurance claims resulting from lightning damage in Colorado, Utah, and Wyoming in USA during the period from 1987 to 1993 resulted in over \$7 million a year in lightning losses [3]. To reduce the vulnerability of electrical and electronic installations against this phenomenon, transient protection devices were developed and they are continuously subjected to further investigations and improvements [1,2,4-6]. Identification of surge protective devices (SPD), behaviour of SPD under overstress situation and the characterization of overstress features are important to manufacturers and its applications in industrial, military, consumer and medical electronic equipment applications. 
During the five year period of 2007-2011, National Fire Protection Association estimates that U.S. local fire departments responded to an estimated average of 22,600 fires started by lightning per year. These fires caused an estimated average of nine civilian deaths, 53 civilian injuries and $\$ 451$ million in direct property damage per year [7].

In the literature there are several studies have been disclosed response of SPD under different transient conditions such as fast current impulses, repetitive current impulse environment and high current derivative impulses [1,2,4-6]. The majority of the experiments reported in the literature have been performed under $8 / 20 \mu$ s (under-short circuit conditions or low-impedance loads) and 1.2/50 $\mu$ s (under-open circuit conditions or high-impedance loads) standard overstress test waveforms [8]. However if the load is not specified or under nonlinear load it is desirable to combine $1.2 / 50 \mu$ s and $8 / 20 \mu$ s waveforms into a single generator as described in [8,9]. ANSI C62.41-1980 suggests that a representative level for maximum overstress on low voltage power system (LVPS) mainly for major feeders and short branch circuits inside a building is given by the combination of $1.2 / 50 \mu$ s waveform with a peak open-circuit of $6 \mathrm{kV}$ and an $8 / 20 \mu$ s waveform with a peak short-circuit of $3 \mathrm{kA}[8,10]$.

On the other hand, protection from surge currents can be attained by either; blocking or limiting them with a large series impedance, or diverting them with a small shunt impedance [8]. Therefore it is desirable to use surge protection components or devices that have a nonlinear V-I relation as for protection circuits [8]. In that way one can have devices that present a small series impedance or large shunt impedance during normal system operation, which is desirable to minimize the effect of the protective circuit on normal system operation [8]. However, during surges the nonlinear elements have a large series impedance or small shunt impedance, which is desirable to effectively block or divert the surge. The use of highly nonlinear components enables effective surge protective circuits to be designed that have minimal effect on normal system operation [8].

During the product testing of these nonlinear surge protective devices, usually measure current and voltage signatures to determine the V-I characteristic curves, and from analysis of these data, parameters for the SPD model are determined. Surge generators with RLC pulse shaping networks are the most common type of surge generators for both standard test waveforms, $1.2 / 50 \mu$ s voltage impulse $\& 8 / 20 \mu$ s current impulse waveforms [8]. The design of a pulse shaping network for the combination waveforms $1.2 / 50 \mu$ s voltage impulse $\& 8 / 20$ $\mu$ s current impulse is described in [11]. Mainly, three different surge generator with RLC pulse shaping networks were used for testing of the characteristics of SPD as per the available literature namely Capacitor Bank generator, Combination waveform generator and commercially available Schaffner generator [1,2,4-6,12-15].

As explained in $[5,6,8,15]$, carryout computer simulations with the help of available SPD models are common practice nowadays in order for testing and studying the characteristics of SPDs. In order for simulating transient response of a surge protection devices, there are two ways which can inject standard overstress test waveforms as the impulse source, couple to the SPD model circuit [8]. One option is to modelling the impulse generator as a circuit model as per the available literature [1,2,4-6,12-15] for both $1.2 / 50 \mu \mathrm{s} \&$ $8 / 20 \mu$ s impulse waveforms.

Second option is to use two analytical equations as explained in $[8,16]$ and recommended by international standards (IEC 60-2, ANSI/IEEE Std 4-1978, and ANSI C62.1-1984) as the source for both $1.2 / 50 \mu$ s voltage impulse $\& 8 / 20 \mu$ s current impulse. 
However, as pointed out in [8], major disadvantage of use of generators with pulse shaping networks is that the load affects the waveshape. The characteristics of the generated waveforms are varied by the impedance of the device that is connected to the generator Therefore same scenario should be there when generator circuit models used for simulation purposes. Nevertheless, when recommended analytical equations used as sources, there cannot be loading effect comes into the picture. Nowadays for SPD simulation studies are based on either with generator models or use analytical equations as sources mostly depending on the researches choice. This is mainly due to lack of guidelines to choose the impulse generator source model. However, to develop a guideline or a recommendation, it is very much essential to know the behaviour of these three generator circuit models under nonlinear surge protecting devices as its load and to be compared with the behaviour under when analytical equations are used as sources.

Therefore, the response of low voltage surge protective devices against different circuit models should be studied. For this reason, the present study was performed in order to understand how a nonlinear load affects the characteristics of the RLC pulse shaping surge generator waveforms under simulation studies. The obtained results were compared with the behavior of the nonlinear load when analytical equations are used as sources for both 1.2/50 $\mu$ s $\& 8 / 20 \mu$ impulses.

\section{SIMULATION SETUP}

All the simulation work was done using the Alternative Transients Program (ATP). The Alternative Transients Program to the Electro-Magnetic Transients Program (ATP-EMTP) is a non-commercial circuit simulation software working in time domain. It was developed for use in the high voltage power system calculations as well as for electronic circuit simulations. The ATP-EMTP has the facility of developing circuit models. Current and voltage sources also can be developed as modelled circuit blocks based on corresponding analytical equations.

On ATP-EMTP platform, a simulation setup was modelled which consist of a transients impulse generator together with a variable impedance load to represent the nonlinear SPD. Evoking the nonlinear SPDs have a large series impedance or small shunt impedance during its operation, two impedance ranges were selected under voltage and current impulses. For voltage impulses, impedance of the system was varied $10 \Omega$ to $10^{6} \mathrm{k} \Omega$ and for current impulses it was between $10^{-4} \Omega$ to $1 \Omega$. For both cases generators were charged to $6.6 \mathrm{kV}$ and the fictive impedance of the generator, which is the ratio between an open circuit voltage peak and a short circuit current peak, was $2 \Omega$ for current impulses and it was $12 \Omega$ for voltage impulses. Blok diagram of the simulation setup is shown in Figure 1 and V \& I probes were introduced to measure voltage across the load and the current trough the load, with the help of ATP-EMTP tools.

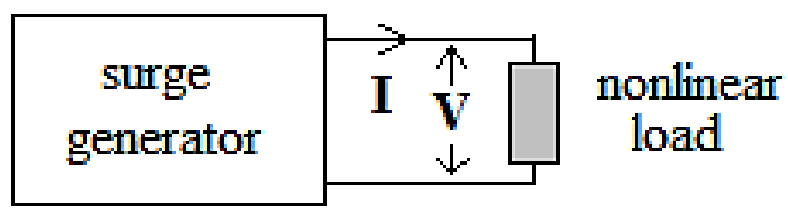

Figure 1. Blok diagram of the simulation setup. 
For this study three generator circuit models available in literature were implemented namely; Capacitor Bank generator, Combination waveform generator and commercially available Schaffner generator [1,2,4-6,12-15] and analytical equations described in $[8,16]$ were modelled as the control sources for both $1.2 / 50 \mu$ s $\& 8 / 20 \mu$ s impulses.

\section{IMPULSE SOURCE MODELS}

The objective of this study was to understand how a nonlinear load affects the characteristics of the impulse generator waveforms in simulation studies. Therefore generator models also have to be included in the computer simulation of this study.

Therefore three ATP-EMTP circuit models were created to implement Capacitor Bank generator, Combination waveform generator and Schaffner generator sources in this study. As the fourth generator, analytical equations were modelled as impulse sources for both $1.2 / 50 \mu \mathrm{s} \& 8 / 20 \mu \mathrm{s}$ impulses. Figure 2 shows two tested impulses for both $1.2 / 50 \mu$ s open circuit voltage waveform and $8 / 20 \mu$ s short circuit current waveform generated using analytical equations.

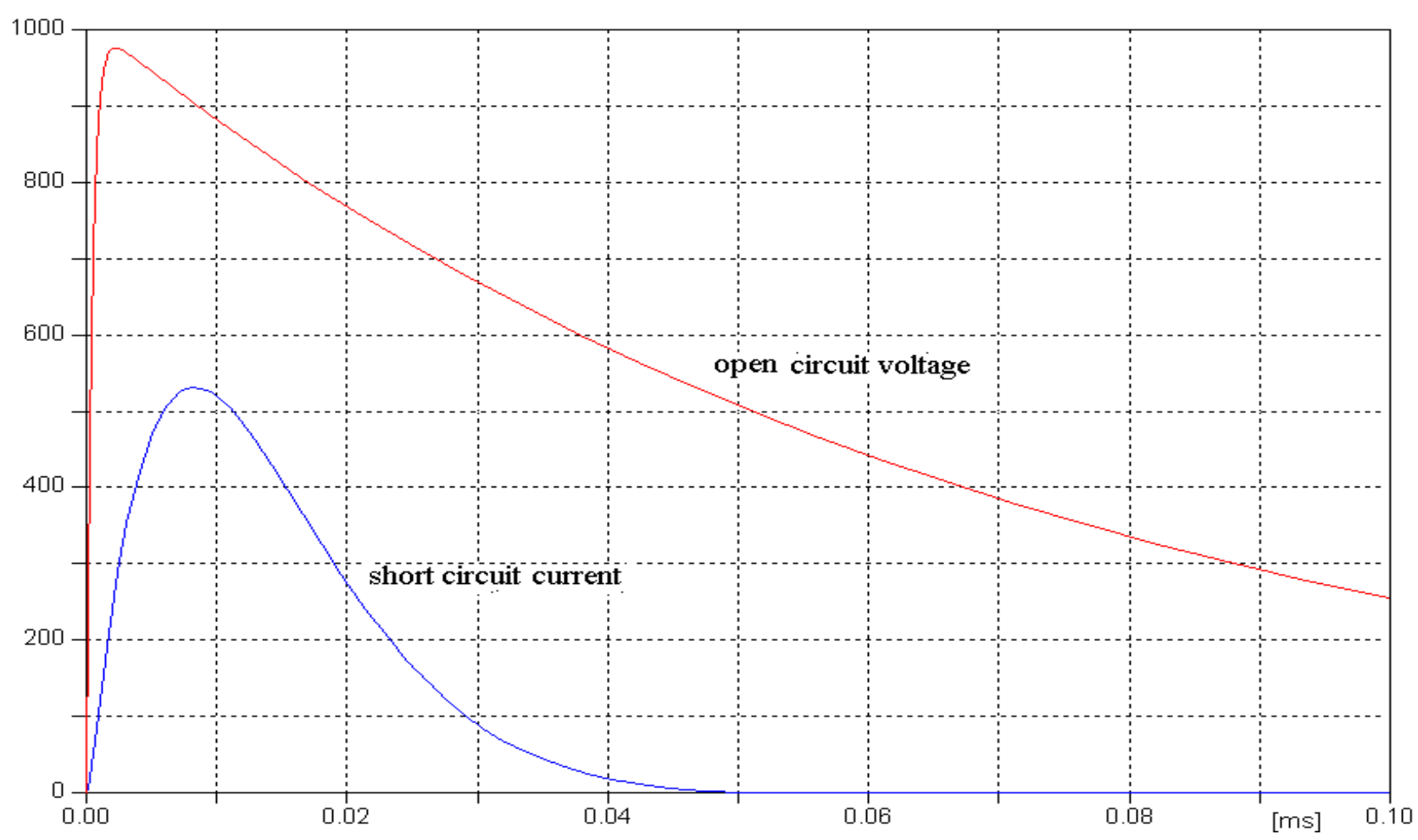

Figure 2. Waveforms for $1.2 / 50 \mu$ s open circuit voltage and $8 / 20 \mu$ s short circuit current.

\section{1. Impulse Generator circuit model 1: Capacitor Bank generator}

As explained in $[12,13]$, an impulse test generator circuit, named Capacitor Bank generator, can be constructed and modelled in ATP-EMTP, using a RLC circuit. Developed circuit model for Capacitor Bank generator is shown in Figure 3. 


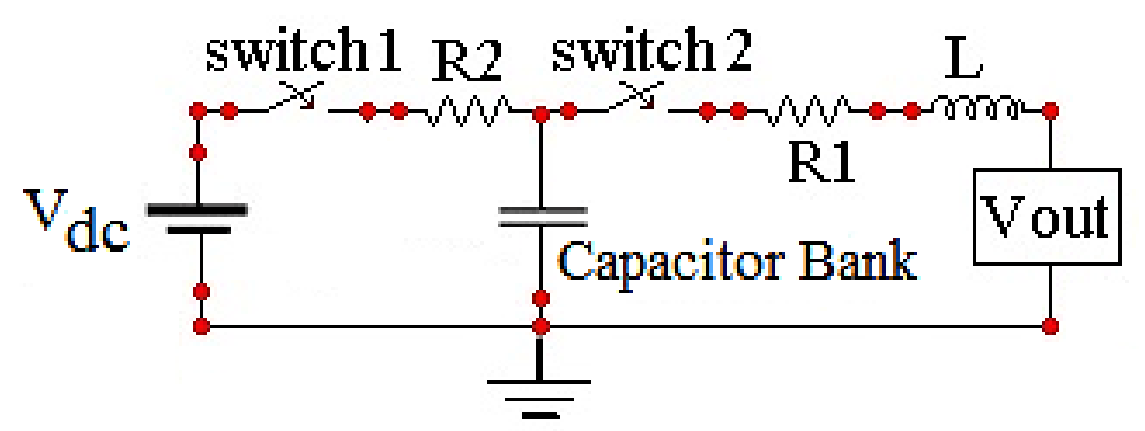

Figure 3. Circuit model for Capacitor Bank generator (parametric values: $R_{1}-0.042 \Omega$, capacitor bank $-235 \mu \mathrm{F}$ and $-0.3 \mu \mathrm{H}[14,15])$.

In this circuit model, a capacitor bank represented by a capacitor will be charged to a specified voltage $\left(\mathrm{V}_{\mathrm{dc}}\right)$, through $\mathrm{R}_{2}$. Then the capacitor will be discharged through a waveshaping $\mathrm{RL}$ network, with $\mathrm{R}_{1}$ being the circuit resistance and $\mathrm{L}$ being the lead inductance due to connections.

\section{2. Impulse Generator circuit model 2: Combination waveform generator}

An impulse test generator circuit, labelled Combination waveform generator, can be constructed and modelled in ATP-EMTP, using a RLC circuit which have been used extensively to achieve a double exponential waveform cited in [12,13]. Circuit model for Combination waveform generator is shown in Figure 4. A bank of capacitors is charged to a particular dc voltage, and then suddenly discharged into an RL waveshaping network by closing the switch. The discharge voltage $\mathrm{V}_{\text {out }}$ gives rise to the desired double exponential waveform.

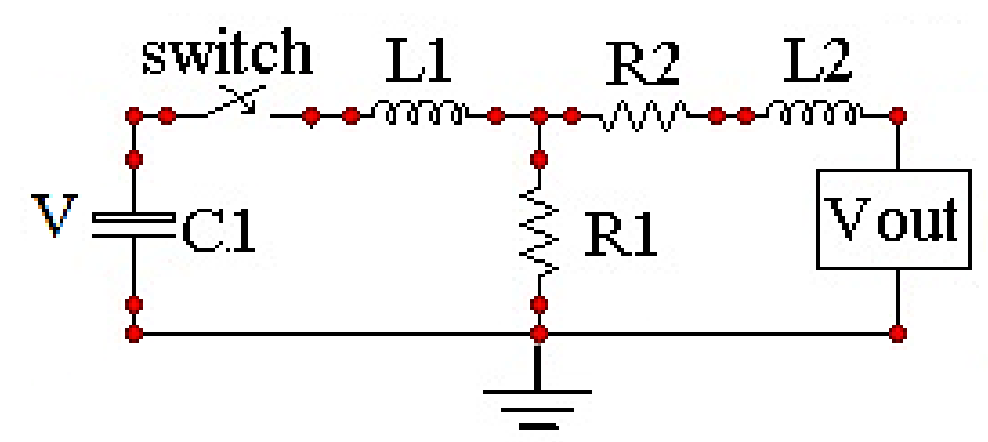

Figure 4. Circuit model for Combination waveform generator (parametric values: $C_{1}-12.5 \mu F, R_{1}$ $5.83 \Omega, \mathrm{R}_{2}-1.3 \Omega, \mathrm{L}_{1}-2.45 \mu \mathrm{H}$ and $\left.\mathrm{L}_{1}-3.15 \mu \mathrm{H}[15]\right)$.

\section{3. Impulse Generator circuit model 3: Schaffner generator NSG650}

The model for Schaffner generator NSG650 was created using the manufacturer specifications. The generator can give a $1.2 / 50 \mu$ s impulse voltage in open circuit and $8 / 20 \mu \mathrm{s}$ impulse current in a short circuit. The modelled circuit for Schaffner generator is shown in Figure 5. 


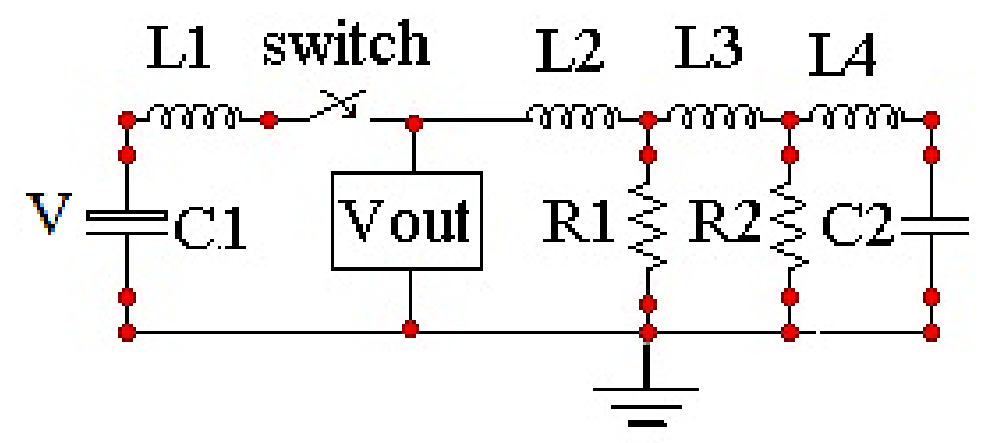

Figure 5. Schaffner generator circuit model (parametric values: $C_{1}-10 \mu F, C_{2}-10 p F, R_{1}-13 \Omega, R_{2}$ $200 \Omega, \mathrm{L}_{1}-12.5 \mu \mathrm{H}, \mathrm{L}_{2}-0.03 \mathrm{mH}, \mathrm{L}_{3}-0.05 \mathrm{mH}$ and $\left.\mathrm{L}_{4}-0.05 \mathrm{mH}[15]\right)$.

\section{4. Impulse Generator model using Analytical equations}

As mentioned earlier two analytical equations recommended by international standards as the simple mathematical relation that describes for both $1.2 / 50 \mu$ s $\& 8 / 20 \mu$ s impulses were implemented in the ATP-EMTP circuit simulation program using its MODELS language. These two models act as self-regulating generator sources which no changes for the characteristics of the waveform occur due to its load connected.

\section{4. 1. Analytical equation for $8 / 20 \mu$ s current waveform}

International standards (IEC 60-2, ANSI/IEEE Std 4-1978, and ANSI C62.1-1984) define $8 / 20 \mu$ s current waveform as a lightning current impulse $[8,16]$. A simple, approximate mathematical expression for $8 / 20 \mu$ s short circuit current waveform that is specified in IEC 60-2, ANSI/IEEE Std 4-1978, and ANSI C62.1-1984 is $\boldsymbol{I}(\boldsymbol{t})$, is given by equation $1[8,16]$.

$$
I(t)=A I_{P} t^{3} \exp (-t / \tau)
$$

where,

$t$ is the time in $\mu$ s $(\mathrm{t} \geq 0)$,

$I_{P}$ is the peak value of current $I(t)$,

$\boldsymbol{A}=0.01243(\mu \mathrm{s})^{-3}, \quad$ and $\quad \tau=3.911 \mu \mathrm{s}$.

\section{4. 2. Analytical equation for $1.2 / 50 \mu$ soltage waveform.}

The $1.2 / 50 \mu$ s waveform is specified in IEC 60-2, ANSI/IEEE Std 4-1978, and ANSI C62.1-1984 [8,16]. A double exponential mathematical relation that describes 1.2/50 $\mu$ s open circuit voltage waveform $\boldsymbol{V}(\boldsymbol{t})$, is given by equation $2[8,16]$.

$$
V(t)=A V_{P}\left\{1-\exp \left(-t / \tau_{1}\right)\right\} \exp \left(-t / \tau_{2}\right)
$$

where,

$t$ is the time in $\mu \mathrm{s}(\mathrm{t} \geq 0)$,

$\boldsymbol{V}_{\boldsymbol{P}}$ is the peak value of voltage $V(t)$,

$\boldsymbol{A}=1.037, \quad \tau_{1}=0.4074 \mu \mathrm{s}, \quad$ and $\quad \tau_{2}=68.22 \mu \mathrm{s}$. 


\section{SIMULATION RESULTS AND ANALYSIS}

Figure 6 shows the measured V-I characteristics across the load impedance connected to four generator sources discussed above simulated with generator charging voltage of 6.6 $\mathrm{kV}$. Figure 6(a) \& (b) show the waveform features of the measured voltage across the load impedance when it was assigned $10^{6} \Omega$ and $10 \Omega$ respectively. Figure 6(c) \& (d) show the waveform features of the measured current through the load impedance when it was assigned $10^{-4} \Omega$ and $1 \Omega$ respectively. As per the waveform features showing in Figure 6(a) \& (b), it is obvious that when load impedance is very large $\left(10^{6} \Omega\right)$, all four generator sources could drive the output close to its open circuit voltage signatures. However when impedance is low $(10 \Omega)$, output voltage is varying from $1.2 / 50 \mu$ s features other than for the generator source model developed using the analytical equation given in (2). As shown in Figure 6(c) \& (d), the measured current through the load impedance showing different characteristics which varies from the short circuit current waveform properties.

Table 1. Measured waveform characteristics of output voltage signatures.

\begin{tabular}{|c|c|c|c|c|c|c|c|c|c|c|c|c|}
\hline \multirow{2}{*}{ 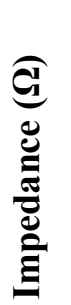 } & \multicolumn{3}{|c|}{$\begin{array}{c}\text { Capacitor bank } \\
\text { generator }\end{array}$} & \multicolumn{3}{|c|}{$\begin{array}{c}\text { Combination } \\
\text { generator }\end{array}$} & \multicolumn{3}{|c|}{$\begin{array}{l}\text { Schaffner } \\
\text { generator }\end{array}$} & \multicolumn{3}{|c|}{ Model equation } \\
\hline & 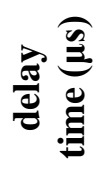 & 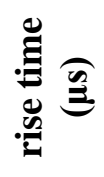 & $\sum_{i} \frac{\widehat{\Omega}}{\Xi}$ & 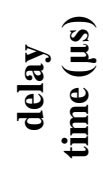 & 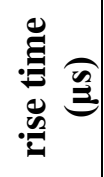 & $\sum_{i} \frac{\sigma}{\xi}$ & 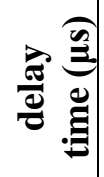 & 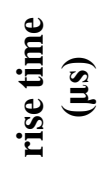 & $\sum_{i=} \frac{\partial}{\Xi}$ & 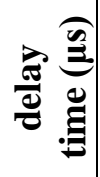 & 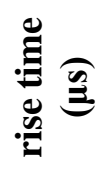 & $\sum_{i}$ \\
\hline 10 & 3.48 & & & 3.63 & & & 3.21 & 2.4 & 166 & 4.95 & 1.2 & \\
\hline 40 & 4.37 & 1.4 & 115 & 4.65 & 1.44 & 99.7 & 4.23 & 1.6 & 112 & 4.95 & 1.20 & 81.8 \\
\hline $10^{2}$ & 4.67 & 1.28 & 88.5 & 4.99 & 1.33 & 90.4 & 4.54 & 1.47 & 99.7 & 4.95 & 1.20 & 81.8 \\
\hline $10^{3}$ & 4.89 & 1.21 & 82.6 & 5.23 & 1.26 & 85.9 & 4.74 & 1.36 & 92.4 & 4.95 & 1.20 & 81.8 \\
\hline $10^{4}$ & 4.92 & 1.21 & 82.2 & 5.25 & 1.26 & 85.5 & 4.76 & 1.35 & 91.6 & 4.95 & 1.20 & 81.8 \\
\hline $10^{5}$ & 4.93 & 1.21 & 82.1 & 5.27 & 1.24 & 85.1 & 4.79 & 1.34 & 91.2 & 4.95 & 1.20 & 81.8 \\
\hline $10^{6}$ & 4.94 & 1.21 & 82.0 & 5.28 & 1.23 & 84.8 & 4.81 & 1.32 & 91.1 & 4.95 & 1.20 & 81.8 \\
\hline
\end{tabular}

Table 2. Measured waveform characteristics of output current signatures.

\begin{tabular}{|c|c|c|c|c|c|c|c|c|c|c|c|c|}
\hline \multirow{2}{*}{ 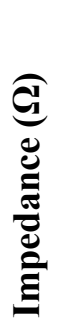 } & \multicolumn{3}{|c|}{$\begin{array}{l}\text { Capacitor bank } \\
\text { generator }\end{array}$} & \multicolumn{3}{|c|}{$\begin{array}{c}\text { Combination } \\
\text { generator }\end{array}$} & \multicolumn{3}{|c|}{$\begin{array}{l}\text { Schaffner } \\
\text { generator }\end{array}$} & \multicolumn{3}{|c|}{ Model equation } \\
\hline & 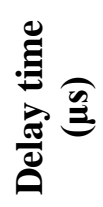 & 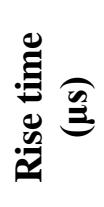 & $\sum_{i}^{\infty}$ & 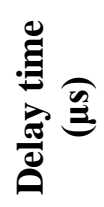 & 氖 & $\sum_{i}^{\infty}$ & 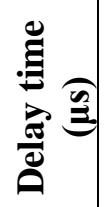 & 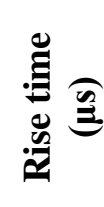 & $\sum_{i}$ & 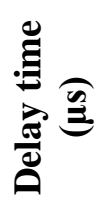 & 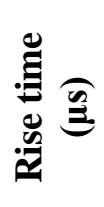 & 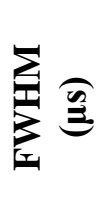 \\
\hline $10^{-4}$ & 18.2 & & & 18.3 & & & 22.0 & 7.30 & 24.5 & 16.2 & 8.02 & 20.7 \\
\hline $10^{-3}$ & 18.6 & & & 18.2 & & & 22.0 & 7.30 & 24.9 & 16.2 & & 20.7 \\
\hline $10^{-2}$ & 19.1 & 6.82 & 23.4 & 18.3 & 5.85 & 20.8 & 22.1 & 7.28 & 25.4 & 16.2 & 8.02 & 20.7 \\
\hline $10^{-1}$ & 22.9 & 5.38 & 24.6 & 18.4 & 5.69 & 22.4 & 22.3 & 7.11 & 27.3 & 16.2 & 8.02 & 20.7 \\
\hline $10^{0}$ & 29.1 & 4.11 & 27.3 & 20.7 & 4.48 & 25.6 & 26.3 & 5.74 & 28.9 & 16.2 & 8.02 & 20.7 \\
\hline
\end{tabular}



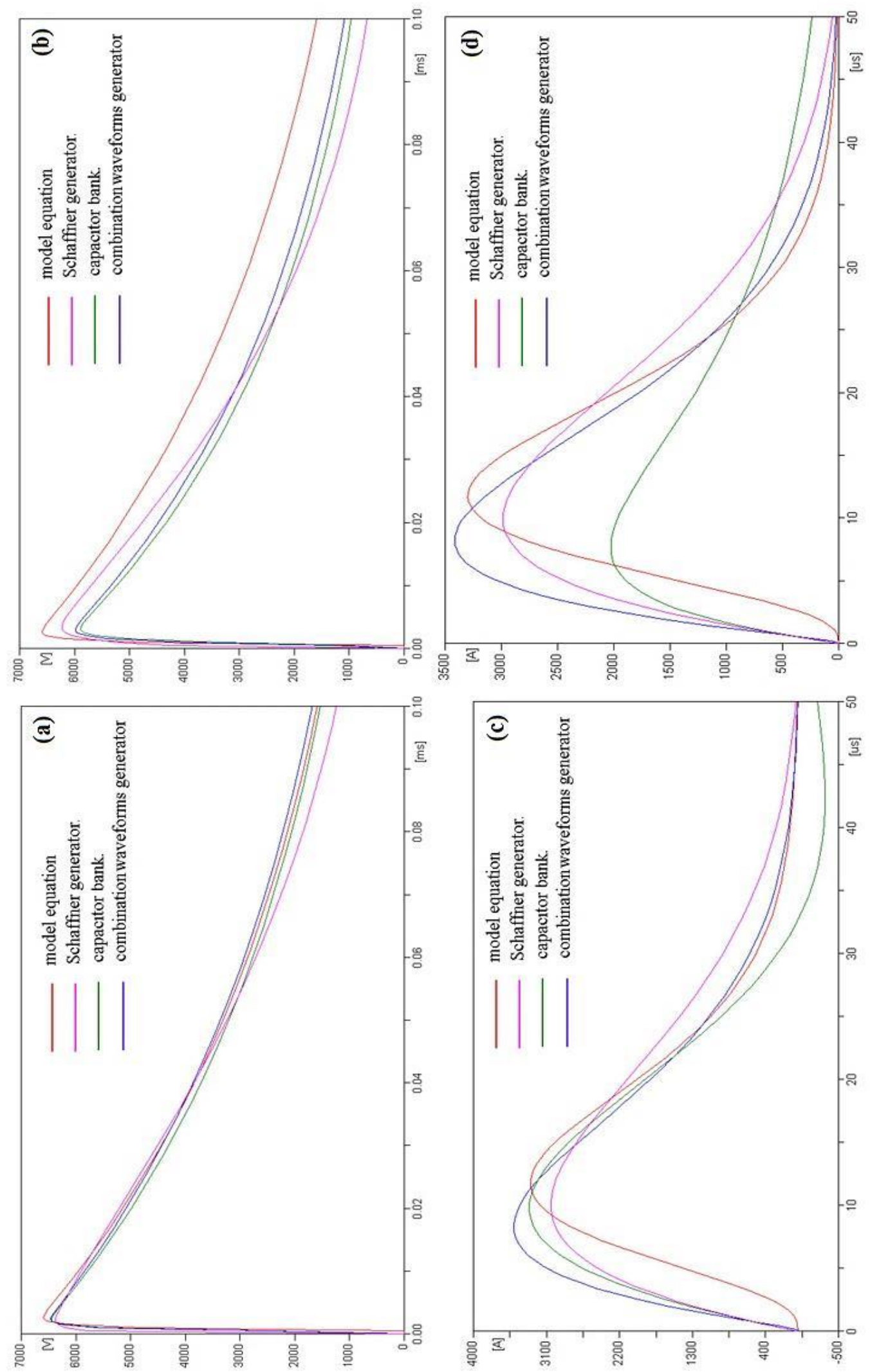

Figure 6. Measured V-I characteristics for the four generator sources with charging voltage of $6.6 \mathrm{kV}$ (a) voltage at $10^{6} \Omega$, (b) voltage at $10 \Omega$, (c) current at $10^{-4} \Omega$, (d) current at $1 \Omega$. 

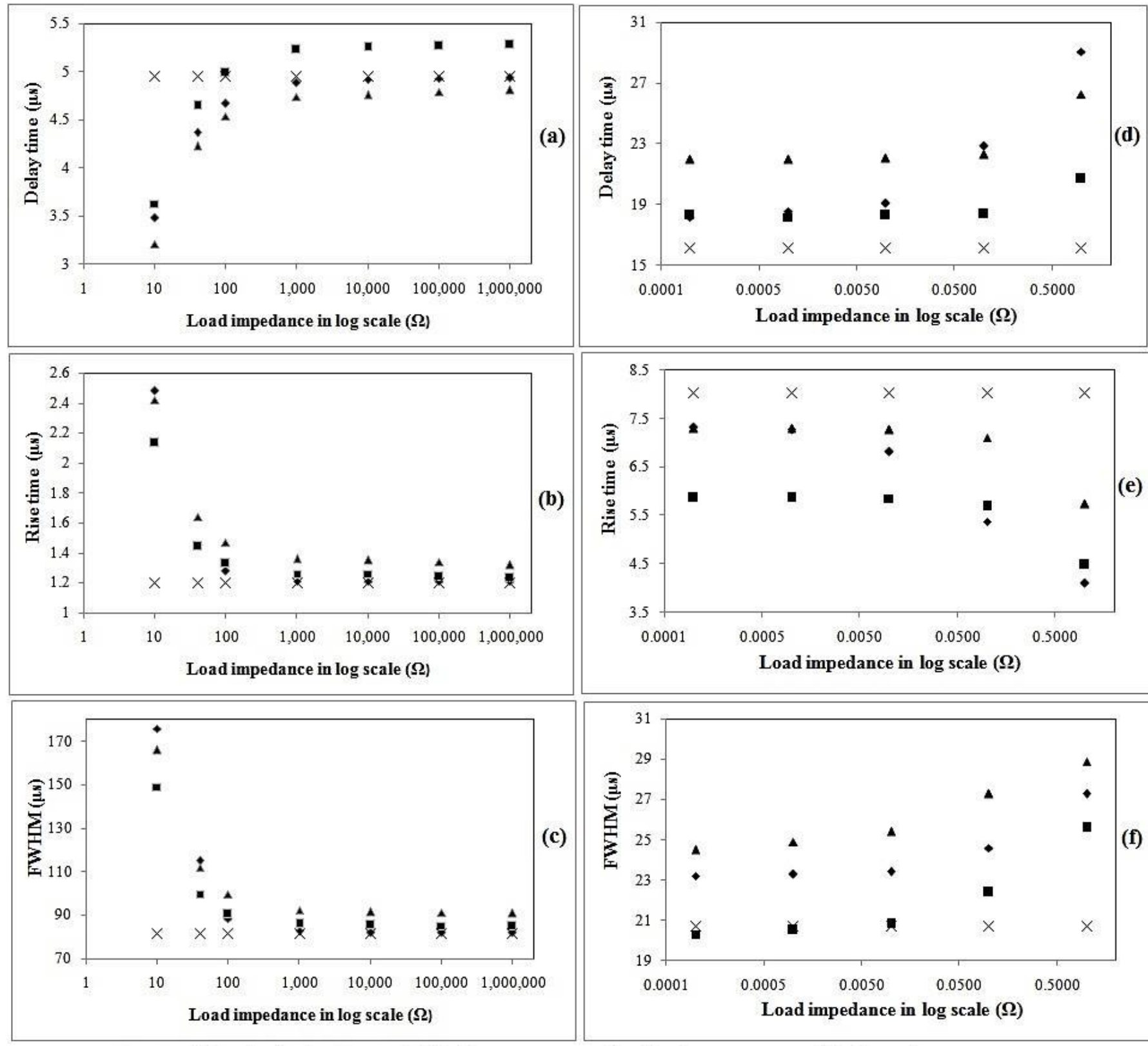

- Capacitor bank

$\Delta$ Schaffner generator

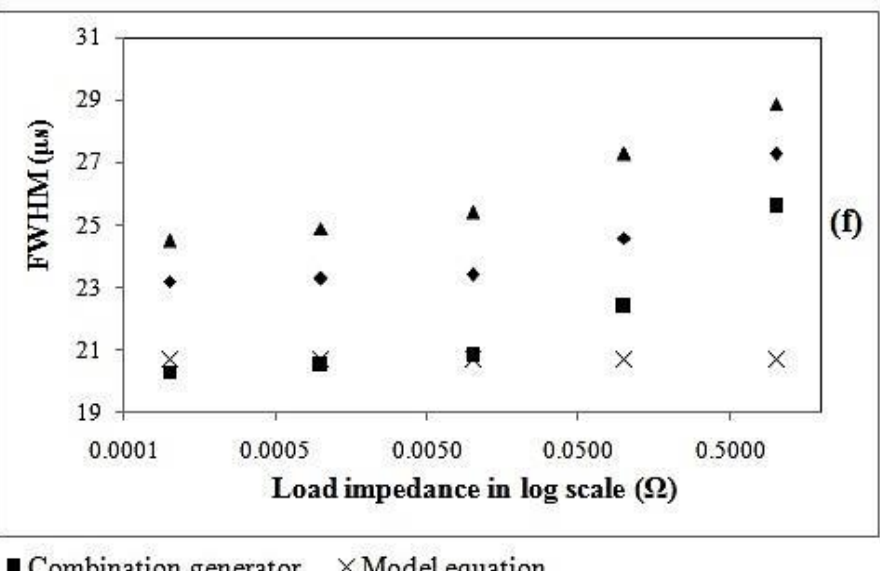

Figure 7. Characteristics of output V-I signatures under four generator sources at the charging voltage of $6.6 \mathrm{kV}$ (a), (b) \& (c) for voltage signatures and (d), (e) \& (f) for current signatures.

When it was assigned very small impedance $\left(10^{-4} \Omega\right)$ its properties were somewhat close towards short circuit conditions and if it increases towards $1 \Omega$ properties deviated from the short circuit condition other than for analytical equation given in (1). Therefore in general, as it can be observed from Figure 6, the characteristics of the generated output waveforms are varied by the impedance of the device that is connected to the generator. When it is connected for a voltage generator with lower impedance and for a current generator with higher impedance above scenario takes place. The above four generator sources were giving output wave shapes with the different waveform characteristics mainly rise time and full width at half-maximum (FWHM). It was also noted that the voltage peak occurs before the current 
peak with a noticeable delay time. If the generator is purely resistive, both current and voltage peaks would have occurred at the same time.

Table 1 and Table 2 shows the tabulated summary of the measured waveform characteristics of output voltage and current signatures respectively for different impedance levels as the load with the charging voltage of $6.6 \mathrm{kV}$. As it can perceive from both Table 1 and Table 2, delay time, rise time and FWHM remain unchanged when used model equations as generator sources for both voltage and current impulses. This implies the behaviour of these generator sources in ideal manner and is independent of the load impedance. However scenario is totally different when used the source models based on Capacitor Bank generator, Combination waveform generator and Schaffner generator.

Graphical overview of measured waveform characteristics; delay time, rise time and FWHM for both voltage and current impulses tabulated in Table 1 and Table 2 are shown in Figure 7. Graphical overview of delay time, rise time and FWHM of output voltage signatures for different load impedances under four generator sources at the charging voltage of $6.6 \mathrm{kV}$ is shown in Figure 7 (a), (b) \& (c) and Figure 7 (d), (e) \& (f) show the same information for the output current signatures.

\section{DISCUSSION}

When simulating the transient response of SPDs with highly nonlinear behaviour, there are many ways which can couple the impulse source into the model circuit. One option is to use the circuit model of the impulse source (mainly RLC pulse shaping surge generators) as indicated in $[1,2,5,6,15]$. However as discussed in $[1,2,4-6,8,15,16]$ analytical equations were developed with international standard guidelines which can be use as the impulse sources. The main objective of this study was to understand how a nonlinear load affects the characteristics of the impulse waveforms under simulation environment. The study was focused to most common type of surge generators currently in use for both standard test waveforms, $1.2 / 50 \mu$ s voltage impulse $\& 8 / 20 \mu$ s current impulse waveforms [8,11]. Three circuit models; Capacitor Bank generator, Combination waveform generator and Schaffner generator were evaluated under nonlinear loads varied from $10 \Omega$ to $10^{6} \Omega$ for voltage impulses and for current impulses it was from $10^{-4} \Omega$ to $1 \Omega$. These two impedance ranges were selected in order to simulate the system load together with a small series impedance and large shunt impedance which are desirable to effectively block or divert the surge with the help of highly nonlinear SPDs as mentioned in [8].

It was found that, for these two load impedance ranges, out of many waveform characteristics, there were three key features formulate obvious significant variations to the both $1.2 / 50 \mu \mathrm{s} \& 8 / 20 \mu$ s impulses. Hence, in this study analysis were carried out to explore such three waveform characteristics; delay time, rise time \& FWHM which are tabulated in Table 1 and Table 2.

When the analytical equations are used as an impulse source it works as a perfect generator and output waveform characteristics are not dependent on the impedance of the system. As tabulated in Table 1 and Table 2 and shown in Figure 7 (a), (b), (c), (d), (e) \& (f) it can confirm that waveform characteristics; delay time, rise time \& FWHM remain unchanged for two impedance ranges considered when used model equations as generator sources for both voltage and current impulses. It was found that as given in Table 1, for $1.2 / 50 \mu$ s voltage impulses, delay time, rise time \& FWHM values were $4.95 \mu \mathrm{s}, 1.20 \mu \mathrm{s}$ and $81.8 \mu$ s respectively throughout the tested impedance range. As given in Table 2 , for $8 / 20 \mu \mathrm{s}$ 
current impulses, these values were $16.2 \mu \mathrm{s}, 8.02 \mu$ s and $20.7 \mu$ s respectively throughout the tested impedance range. Hence, characteristics of waveforms introduced to the system are not depending on the impedance of the system and no loading effects in action under this scenario. Thus when analytical equations are used as impulse sources, both voltage and current source models were performed as ideal generators.

However stability of waveform characteristics is differ with other three generator source models; Capacitor Bank generator, Combination waveform generator and Schaffner generator. In the case of Capacitor Bank generator, for voltage impulses, delay time, rise time \& FWHM values were within the range of 3.48-4.94 $\mu \mathrm{s}, 2.48-1.21 \mu \mathrm{s}$ and $176-82.0 \mu \mathrm{s}$ respectively. For current impulses these values were within the range of 18.2-29.1 $\mu \mathrm{s}, 7.33$ $4.11 \mu \mathrm{s}$ and 23.2-27.3 $\mu$ s respectively. In the case of Combination waveform generator, for voltage impulses, delay time, rise time \& FWHM values were within the range of 3.63-5.28 $\mu \mathrm{s}, 2.13-1.23 \mu \mathrm{s}$ and $149-84.8 \mu \mathrm{s}$ respectively. For current impulses these values were within the range of 18.3-20.7 $\mu \mathrm{s}, 5.87-4.48 \mu$ s and 20.3-25.6 $\mu$ s respectively. In the case of Schaffner generator, for voltage impulses, delay time, rise time \& FWHM values were within the range of 3.21-4.81 $\mu \mathrm{s}, 2.42-1.32 \mu \mathrm{s}$ and 166-91.1 $\mu \mathrm{s}$ respectively. For current impulses these values were within the range of 22.0-26.3 $\mu$ s, 7.30-5.74 $\mu$ s and 24.5-28.9 $\mu$ s respectively.

Therefore it was obvious that, waveform characteristics of the generated impulses are varied by the influence of the nonlinear load impedance. When it was connected for a voltage generator with lower impedance or for a current generator with higher impedance these variations takes place. These results implies that the impedance of the system load be able to influence the generator characteristics. When impedance of the nonlinear load increased, characteristics of current impulse waveform were also changed and significantly deviated from the expected values.

However when the impedance is at its lowest value, these parametric values were almost equal to the values obtained when the analytical equation was used as current impulse source. When impedance of the nonlinear load decreased, characteristics of voltage impulse waveform were also deviated from the expected values. However when the impedance is at its highest value, these parametric values were almost equal to the values obtained when the analytical equation was used as voltage impulse source. Figure 7 (a), (b), (c), (d), (e) \& (f) show the graphical representation of these deviations.

Figure 8 shows the amount of absolute deviation of delay time, rise time \& FWHM for the values obtained for Capacitor Bank generator, Combination waveform generator and Schaffner generator with respect to the simulations using analytical equations. As it can be observed, in general these deviations were at its minimum when the system impedance is $10^{6}$ $\Omega$ for voltage impulses. For current impulses, these deviations were at its minimum for the system impedance at $10^{-4} \Omega$.

In the case of Capacitor Bank generator, these deviations for voltage impulses were at its maximum of $29.70 \%, 106.67 \%$ \& $115.16 \%$ for delay time, rise time \& FWHM respectively at the system impedance of $10 \Omega$. However, at the system impedance of $10^{6} \Omega$, these deviations were at its minimum of $0.20 \%, 0.83 \% \& 0.24 \%$ respectively. For Combination waveform generator, these deviations were at its maximum of $26.67 \%, 77.50 \%$ $\& 82.15 \%$ respectively at the system impedance of $10 \Omega$. However, at the system impedance of $10^{6} \Omega$, these deviations were at its minimum of $6.67 \%, 2.50 \% \& 3.67 \%$ respectively. In the case of Schaffner generator, these deviations were at its maximum of $35.15 \%, 101.67 \%$ $\& 102.93 \%$ respectively at the system impedance of $10 \Omega$. However, at the system impedance of $10^{6} \Omega$, these deviations were at its minimum of $2.83 \%, 10.00 \% \& 11.37 \%$ respectively. 

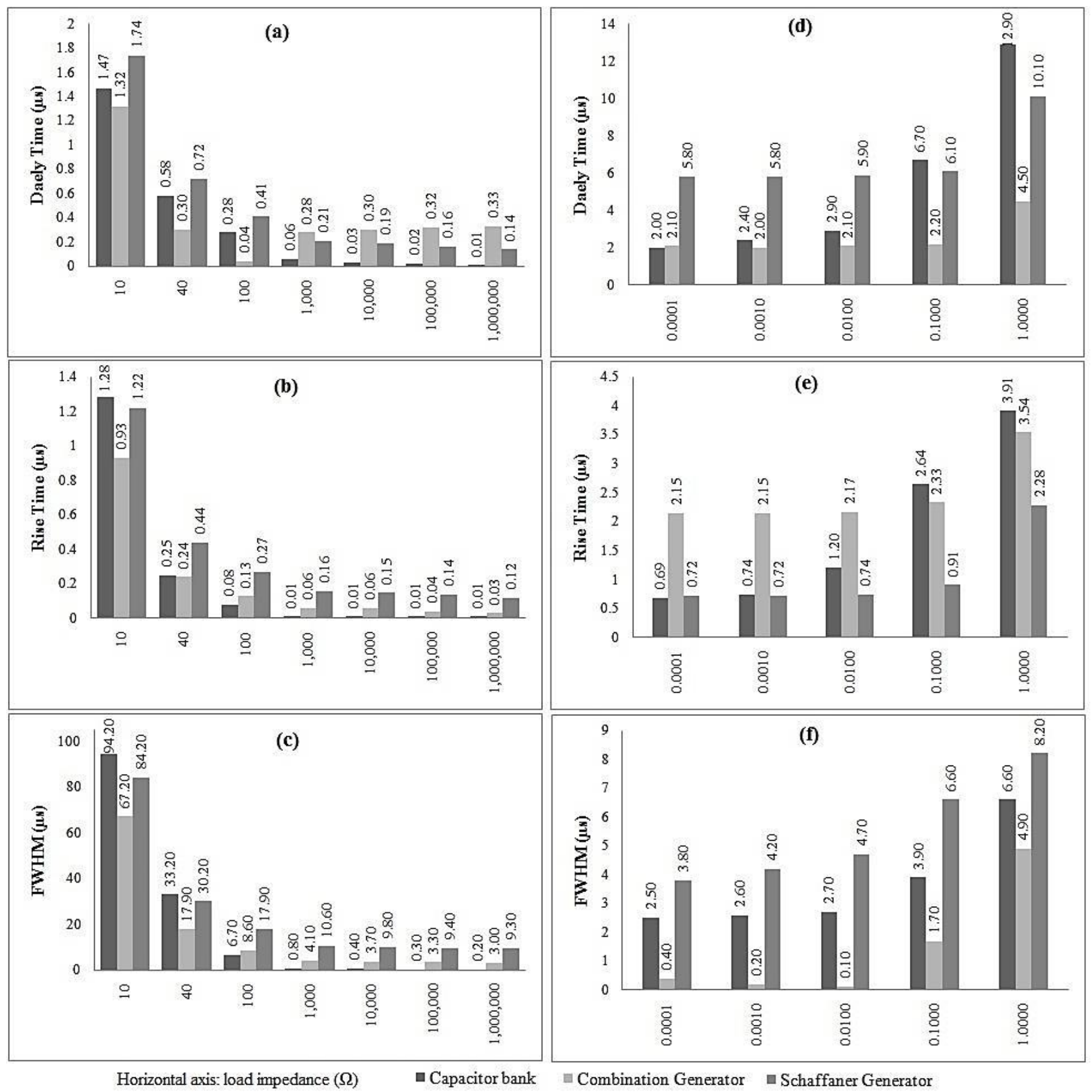

Horizontal axis: load impedance $(\Omega)$

- Capacitor bank

- Combination Generator $\quad$ Schaffaner Generator

Figure 8. Amount of absolute deviation with respect to the analytical equations (a), (b) \& (c) for voltage signatures and (d), (e) \& (f) for current signatures.

In the case of Capacitor Bank generator, these deviations for current impulses were at its maximum of $79.63 \%, 48.75 \% \& 31.88 \%$ for delay time, rise time \& FWHM respectively at the system impedance of $1 \Omega$.

However, at the system impedance of $10^{-4} \Omega$, these deviations were at its minimum of $12.35 \%, 8.60 \%$ \& $12.08 \%$ respectively. For Combination waveform generator, these deviations were at its maximum of $27.78 \%, 41.44 \% \& 23.67 \%$ respectively at the system 
impedance of $1 \Omega$. However, at the system impedance of $10^{-4} \Omega$, these deviations were at its minimum of $12.96 \%, 26.81 \% \& 1.93 \%$ respectively. In the case of Schaffner generator, these deviations were at its maximum of $62.35 \%, 28.43 \% \& 39.61 \%$ respectively at the system impedance of $1 \Omega$. However, at the system impedance of $10^{-4} \Omega$, these deviations were at its minimum of $35.80 \%, 8.98 \%$ \& $18.36 \%$ respectively.

Therefore it can be concluded that these three generator circuit models were giving waveshapes with the different characteristics when open circuited (high impedance load) and short circuited (low impedance load) which shows significant deviations compared to simulations using analytical equations. The deviations were due to influence caused by the nonlinear load to the generator circuit parameters and effective internal impedance. Results of this study show that the characteristics of the generated waveforms are varied significantly by the impedance of the nonlinear load.

On the other hand the main requirement for SPD analysis on simulation platform is that the model should be capable reproducing the waveshapes of voltage across and current through the SPDs realistically. Usually, V-I characteristics of SPDs are reproduced in the model by using nonlinear resistance with some accompanying elementary elements (inductance, capacitance). Most of the previous SPD studies conducted and recommendations generated with the assistance on simulation platform were either using generator circuit models or recommended analytical equations and sometimes had used both approaches $[1,2,4-6,10,15,16]$.

\section{CONCLUSION}

When analytical equations are used as impulse sources it worked as perfect generators and V-I characteristics are not depend on the impedance of the system. However V-I characteristics of the generated waveforms are varied significantly by the impedance of the nonlinear load that is connected to the generator circuit models. The deviations were due to influence caused by the nonlinear load to the generator circuit parameters and effective internal impedance. Therefore it can recommend to be used analytical equations as impulse sources on ATP-EMTP simulation for testing of SPDs. Hence it can study exactly the response of the SPD for recommended impulse waveforms. Nevertheless if the intention of the simulation study is to compare the SPD responses with experimental results, better approach is to use the impulse generator circuit model developed by considering the actual circuit parameters.

\section{Acknowledgements}

Assistance provided by Mr. KKDL Ruvinda is highly acknowledged.

\section{References}

[1] R. Montaño, M. Edirisinghe, V. Cooray, F. Roman, IEEE Transactions on Power Delivery 22(4) (2007) 2185-2190. 
[2] Mahesh Edirisinghe, Raul Montaño, Vernon Cooray, "Response of Surge Protection Devices to Fast Current Impulses," 27th International Conference on Lightning Protection - ICLP, France (September 2004).

[3] R. L. Holle, R. E. López, L. J. Arnol, J. Endres Journal of Applied Meteorology 35(8) (1995) 1344-1351.

[4] Mahesh Edirisinghe, Mahendra Fernando, Vernon Cooray, International Journal of Engineering and Science Research 2(7) (2012) 2185-2190.

[5] Mahesh Edirisinghe, Raul Montaño, Vernon Cooray, F. Roman, International Letters of Chemistry, Physics and Astronomy 6 (2013) 40-53.

[6] Raul Montaño, Mahesh Edirisinghe, Vernon Cooray and Fransisco Roman, "Varistors and Gas Discharge tubes models: a comparison between theory and practice," $27^{\text {th }}$ International Conference on Lightning Protection - ICLP, France (September 2004).

[7] Marty Ahrens, "Lightning Fires and Lightning Strikes," National Fire Protection Association, Fire Analysis and Research Division, pp. 1 (June 2013).

[8] R. B. Standler, "Protection of Electronic Circuits from Overvoltages, John Wiley \& Sons, 1989; pp. 87-91, 109-110, 387-388.

[9] P. Richman, "Single Output, Voltage and Current Surge Generation for Testing Electronic Systems," IEEE 1983 Electromagnetic Compatibility Symposium, pp. 47-51, 1983.

[10] American National Standard C62.41-1980, IEEE Guide for Surge Voltages in LowVoltage AC Power Circuits.

[11] J. Wiesinger, Mess- und Proftechnik 104 (1983) 1102-1105.

[12] M. S. Naidu and V. Kamaraju, "High Voltage Engineering second edition", McGrawHill, New York, 1996.

[13] Cairo University Giza, Egypt, "High Voltage Engineering, Theory and Practice, Edited by M. Khalifa”, Marcel Dekker, Inc., 1990.

[14] Osama Mohammed and Roberto Siegert, "Evaluation of a Hybrid Surge Testing Generator Configuration using Computer Based Simulations", Southeastcon '99. Proceedings IEEE, 193-196, 1999.

[15] Mihael Zitnik, Numerical modeling of transients in electrical systems, Ph.D. thesis, Uppsala University 2001.

[16] R. B. Standler, IEEE Transactions on Electromagnetic Compatibility 30 (1988) 69-71. 